\section{Uso da avaliação de tecnologias em saúde em processos de análise para incorporação de tecnologias no Sistema Único de Saúde no Ministério da Saúde}

\author{
Use of health technology assessment in \\ decision-making processes by the Brazilian \\ Ministry of Health on the incorporation of \\ technologies in the Brazilian Unified \\ National Health System
}

\section{Evaluación de tecnologías en salud en procesos de análisis para la incorporación de las mismas al Sistema Único de Salud, perteneciente al Ministerio de Salud}

\begin{abstract}
Policies for scientific development and knowledge production in health have increased in recent decades. In Brazil, incentives for research, development, and innovation have been part of the National Health Act since 1990, and science and technology policies for health, including health technology assessment (HTA), have been implemented since 1994, as in many other countries. The emphasis is now on impact evaluation of HTA policies in the incorporation of technologies by health services and systems. The article presents a case study of HTA utilization in decision-making processes in the Brazilian Ministry of Health, analyzing participation by the Department of Science and Technology (DECIT), responsible for the production of assessments used in the Commission on Technology Incorporation (CITEC) of the Ministry of Health from 2008 to 2010. CITEC used 103 assessments in its decisions during this period, of which DECIT produced 80\%. Nearly all were literature reviews on therapeutic technologies. An increase in knowledge production was observed. A methodological and political learning process appears to have occurred in the use of HTA, but its impact on Brazilian Unified National Health System remains unclear
\end{abstract}

Unified Health System; Evaluation Studies; Health Technology Assessment
Hillegonda Maria Dutilh Novaes 1 Flávia Tavares Silva Elias 2

\section{Resumo}

As políticas científicas com objetivos de produção de conhecimentos em saúde foram ampliadas nas últimas décadas. No Brasil, o incentivo à pesquisa, desenvolvimento e inovação em saúde constou na Lei Orgânica da Saúde desde 1990, e políticas científicas e tecnológicas para a área da saúde foram propostas a partir de 1994, incluindo políticas de desenvolvimento da avaliação de tecnologias em saúde (ATS), como em outros paises. Coloca-se agora a avaliação dos impactos da ATS sobre a gestão e incorporação de tecnologias nos sistemas de saúde. Como estudo de caso para o uso da ATS em processos de incorporação de tecnologias no Brasil foi analisada a participação do Departamento de Ciência e Tecnologia (DECIT) do, Ministério da Saúde, na Comissão de Incorporação de Tecnologias do Ministério da Saúde (CITEC), no período 2008-2010. Foram utilizados 103 estudos na CITEC, 80\% produzidos pelo DECIT, a maioria sínteses com base na literatura existente, e $80 \%$ das tecnologias tinham propósito terapêutico, com tendência de crescimento da produção no período. Está em curso um processo de aprendizado tanto metodológico quanto político para o uso da ATS, mas há necessidade de aprofundar a análise do seu impacto sobre o SUS.

Sistema Único de Saúde; Estudos de Avaliação; Avaliação de Tecnologias de Saúde 


\section{Introdução}

Políticas científicas que têm por objetivo apoiar a produção de conhecimentos em saúde ampliaram nas últimas décadas em muitos países, nas suas modalidades, abrangência temática e volume de recursos. Elas se assentam na compreensão de que poderão e deverão contribuir para a qualidade na atenção à saúde, para o desenvolvimento científico e tecnológico direcionado às necessidades de saúde das populações e, quando integradas às políticas de desenvolvimento econômico, produtivo e de inovação, devem apoiar o crescimento econômico do país 1,2. Há também o reconhecimento crescente que para esses impactos da pesquisa se realizarem são necessárias ações que promovam a articulação entre a produção científica e os diferentes setores sociais estratégicos, a fim de realizar esses objetivos, e várias proposições têm sido apresentadas, baseadas em referências teóricas diversas e de diferentes tipos 3,4,5.

No Brasil, o incentivo à pesquisa, desenvolvimento e inovação em saúde consta na Lei Orgânica da Saúde desde 1990, e políticas científicas e tecnológicas específicas para a área da saúde foram iniciadas em 1994 6. A Política Nacional de Ciência, Tecnologia e Inovação em Saúde (PNCTS), formalizada em 2004, incluiu entre as suas estratégias a avaliação de tecnologias em saúde (ATS) como instrumento que contribui para o aprimoramento da capacidade regulatória do Estado na incorporação de tecnologias nos sistemas de saúde.

A avaliação tecnológica na área da saúde, como área de conhecimentos e práticas, teve início nos anos 70 nos Estados Unidos, vinculada às atividades do legislativo americano e a seguir se desenvolveu nos países da Europa Ocidental como parte da gestão dos sistemas de saúde, notadamente naqueles com sistemas de saúde públicos e de cobertura universal (Suécia, Holanda, Reino Unido) 7.

No nosso país, ocorreram pesquisas e encontros pontuais em ATS principalmente no meio acadêmico ao longo dos anos 80 e início dos anos 90. Na segunda metade dos anos 90, o Ministério da Saúde obteve financiamento externo (Banco Mundial e Banco Interamericano de Desenvolvimento - BID) para o fortalecimento do SUS por meio do projeto governamental Reforço à Reorganização do Sistema Único de Saúde (REFORSUS), concentrando-se na aquisição de equipamentos médico-hospitalares e no aprimoramento da gestão e proposta de avaliação dos sistemas e serviços de saúde, incluindo a avaliação tecnológica, por recomendação dos agentes financiadores 8 .
A partir do final da década de 90, o Ministério da Saúde desenvolveu iniciativas para buscar o estabelecimento de políticas de avaliação de tecnologias na sua estrutura regimental 9. Entre 2003 a 2004, foi criado um grupo de trabalho no âmbito do Conselho de Ciência, Tecnologia e Inovação do Ministério da Saúde. Em 2005, foi criada na Secretaria de Ciência e Tecnologia e Insumos Estratégicos (SCTIE), no seu Departamento de Ciência e Tecnologia (DECIT), uma coordenação específica responsável pela implantação e disseminação das ações de ATS no SUS, tanto na produção de conhecimento quanto no uso da gestão em saúde ${ }^{9}$. Em 2008, foi implantada a Rede Brasileira de Avaliação de Tecnologias em Saúde (REBRATS), com o objetivo de aproximar as instituições acadêmicas e os serviços de saúde, para produzir e sistematizar informações necessárias aos processos de tomada de decisão de incorporação de tecnologias no SUS no Ministério da Saúde e em secretarias estaduais e municipais de saúde.

Duas estratégias nortearam a política de implantação do uso dos resultados de estudos em ATS nos processos de gestão de tecnologias no SUS nesse período: a elaboração da Política Nacional de Gestão de Tecnologia em Saúde (PNGTS) e a criação da Comissão de Incorporação de Tecnologia (CITEC) do Ministério da Saúde. A PNGTS foi elaborada por um comitê com representação de múltiplas instâncias políticas e aprovada no Conselho Nacional de Saúde (CNS) e na Comissão de Intergestores Tripartite (CIT). A Comissão de Incorporação foi criada como uma das diretrizes dessa política, com o objetivo de estruturar fluxo para demandas de incorporação, exclusão ou alteração de novas tecnologias no SUS. O propósito era contribuir para a maximização dos benefícios de saúde a serem obtidos com os recursos disponíveis e o acesso da população a tecnologias efetivas e seguras, em condições de equidade 11,12.

Entre 2006 e 2008, a CITEC foi coordenada pela Secretaria de Atenção à Saúde (SAS), e em dezembro de 2008 (Portaria MS/GM no 2.587/2008) foi transferida para SCTIE. Foram definidas como suas competências: recomendar a incorporação ou retirada de produtos de saúde na lista de procedimentos do SUS e do rol de procedimentos da Agência Nacional de Saúde Suplementar (ANS); propor a revisão de diretrizes clínicas e protocolos terapêuticos e assistenciais de interesse para o sistema público de saúde, e solicitar a realização de estudos de ATS para subsidiar as atividades de análise e recomendação.

O papel do DECIT nessa fase, estabelecido em portarias ministeriais, consistia na produção e adoção de providências para o desenvol- 
vimento de estudos com o objetivo de subsidiar as decisões na CITEC. Em 2009, essa função foi formalmente incluída na estrutura regimental do Ministério da Saúde, com a atribuição de coordenar a formulação e implementação de políticas, programas e ações de avaliação de tecnologias no SUS. Desde então, novas alterações ocorreram na estrutura da SCTIE, e em 2011 a CITEC passou a ser denominada Comissão Nacional de Incorporação de Tecnologias (CONITEC), ficando sob a responsabilidade de um novo departamento da SCTIE, Departamento de Gestão e Incorporação de Tecnologias em Saúde (DGTIS).

De acordo com as diretrizes da PNGTS, os processos que regulam a aprovação para o mercado das tecnologias em saúde são de responsabilidade da Agência Nacional de Vigilância Sanitária (ANVISA), que também regula os preços dos medicamentos. As pesquisas e avaliações de interesse público são de responsabilidade do DECIT e os processos que definem a incorporação de tecnologias ocorrem na CONITEC.

O setor público no Brasil procurou dar conta da produção e oferta contínuas de novas tecnologias em saúde que geram forte demanda para sua incorporação no SUS pelos profissionais, gestores e população, em um cenário de recursos financeiros e de gestão sempre insuficientes, sendo responsável por manter um sistema de atenção à saúde efetivo, eficiente e equitativo. A contínua construção e redefinição das instâncias e formas pelas quais o setor público se organizou são semelhantes ao observado em outros países. Desde os anos 80, organizações ou instâncias responsáveis pela realização e uso das avaliações de tecnologias em saúde foram implantadas por países desenvolvidos, com o objetivo de orientar a introdução de intervenções efetivas nos serviços de saúde, contextualizadas aos processos decisórios 13 .

Em anos recentes foram realizadas avaliações dessas organizações, buscando identificar as suas principais características e impactos sobre os processos de incorporação de tecnologias em saúde. Em um estudo de cinco agências bem estruturadas de ATS (DAHTA@DIMDI/Alemanha, IQWiG/Alemanha, HAS/França, NICE/Reino Unido e SBU/Suécia), foram observadas similaridades quanto ao escopo de tecnologias avaliadas, orientações metodológicas, organização dos processos e disseminação dos resultados, e diferenças nos processos de definição de prioridades dos estudos e de decisão para implementação das intervenções avaliadas 14 .

Em estudo 15 que incluiu organizações de países desenvolvidos (Europa, Estados Unidos, Canadá, Austrália) e países em desenvolvimento como a Coreia do Sul, Taiwan e Brasil (ANVISA), e que utilizou como metodologia a verificação da aplicação de princípios de qualidade da ATS propostos pela comunidade científica da área de ATS, observou-se que houve melhor conformidade aos princípios na aplicação dos métodos recomendados, mas grande variabilidade no que se refere aos contextos políticos em que a ATS é utilizada nos processos relativos à incorporação de tecnologias nos sistemas de saúde.

Outro estudo, voltado para a América Latina 16, utilizou esses mesmos princípios e enviou questionários para um grande número de profissionais identificados como produtores de ATS ou gestores de tecnologias. Apenas 10\% responderam, com predomínio de pesquisadores e concentração de respondentes da Argentina e do Brasil, e foram observadas importantes diferenças sobre a compreensão dos princípios e a sua aplicação, com um pouco mais de conformidade aos princípios em respondentes de países que têm instâncias mais estruturadas e produziram recomendações nacionais para estudos em ATS, como Argentina, Brasil, Chile e México.

Estudo com base na revisão da literatura 17 considerou que os programas de ATS na América Latina vêm tentando alcançar os objetivos propostos pela área, porém, destacou que o foco dos documentos produzidos em curto espaço de tempo está nas tecnologias de alto custo, abordadas principalmente nas perspectivas clínica e econômica, sem considerar critérios éticos, organizacionais e legais.

Uma análise das organizações de ATS em 33 países, incluindo o Brasil (DECIT), identificou que as atividades dominantes das agências eram a produção de recomendações sobre medicamentos, considerando efetividade e, em menor escala, custo-efetividade. A maior dificuldade apontada pelas organizações foi a manutenção de recursos financeiros e de pessoal qualificado 18.

A maior parte dos estudos mencionados foi realizada na perspectiva acadêmica e/ou de sistema de saúde. No entanto, também as indústrias produtoras de tecnologias têm realizado estudos do desempenho e impacto de organizações de ATS. Uma empresa de consultoria britânica desenvolveu em 2009 a análise de algumas agências, por encomenda de associações de produtores de medicamentos e imunobiológicos 19 . Foram selecionadas 15 organizações de ATS como estudos de caso, representando países com condições econômicas e políticas distintas, inclusive o Brasil (CITEC). Foi priorizada a análise do seu impacto sobre a incorporação de tecnologias, e os resultados foram variáveis em relação ao tempo entre a aprovação de entrada no mercado e o acesso dos pacientes ao medicamento. A relação 
entre o tipo de estudo e o preço no mercado ou o valor para reembolso, assim como os processos e decisões adotados para as mesmas tecnologias também variaram entre as organizações.

A literatura indica que a ATS como política e atividade de produção de conhecimento tem ampliado nos últimos anos, em particular nos países desenvolvidos, mas que a sua inserção nas políticas de saúde e impacto sobre os sistemas de saúde são ainda variáveis e mais limitados do que os objetivos anunciados 20. Mesmo em países como Reino Unido, França, Holanda e Suécia, a tradução da ATS em políticas tem sido desafiadora e altamente complexa, e a despeito do seu crescimento nas últimas duas décadas, sua influência permaneceu marginal no que se refere a mudanças mais profundas nas definições de prioridades na perspectiva do bem-estar de toda a população.

Considerando que é importante conhecer, em diferentes perspectivas e com maior detalhamento, a implementação de objetivos da PNCTS referentes à produção e utilização de estudos de ATS em processos de gestão de incorporação de tecnologias, apresenta-se a seguir, como estudo de caso, a participação do DECIT nos processos de análise e decisão da CITEC, em período anterior à CONITEC, entre 2008 e 2010.

\section{Estudo de caso: participação do DECIT nos processos de análise e decisão da CITEC, 2008-2010}

Nos anos de 2008 a 2010 a CITEC foi composta por titular e suplente indicados pelas secretarias do Ministério da Saúde e agências reguladoras. Ao DECIT foi atribuída a função de produção dos estudos e apoio às áreas técnicas na análise prévia. Para eleição do tipo de estudo eram considerados a pergunta da avaliação, o tempo disponível para envio do relatório final e a experiência exigida para se realizar a avaliação. A maior ou menor disponibilidade de trabalhos publicados em revistas científicas e a prioridade das áreas técnicas do Ministério da Saúde definiam a ordem de prioridade na elaboração dos estudos.

O fluxo e o mecanismo de avaliação e incorporação de tecnologias no SUS, adotados entre 2008 e 2010, foram os seguintes:

a) As solicitações para incorporação de tecnologias, protocoladas na SCTIE, eram encaminhadas à CITEC;

b) A CITEC fazia a análise prévia das solicitações recebidas e enviava às áreas técnicas responsáveis pelos programas;

c) As áreas técnicas emitiam pareceres concluindo quanto a sua admissibilidade com ba- se na relevância e no impacto da incorporação de tecnologia no SUS, bem como na existência de sólidas evidências científicas de efetividade da tecnologia proposta, podendo contar com o DECIT para essa análise;

d) Havendo conclusão negativa, a Comissão comunicava por ofício, ao demandante, e dava por encerrado o processo;

e) Em caso de a tecnologia ser admissível pelas áreas técnicas, mas necessitasse de estudos de ATS, a Comissão solicitava ao DECIT/SCTIE a realização de estudos de ATS;

f) O DECIT/SCTIE definia as providências necessárias para a produção, contratação, financiamento, desenvolvimento e monitoramento dos estudos de ATS;

g) Os estudos de ATS eram apresentados à medida da necessidade de pauta na CITEC, em fase de produto intermediário ou resultado final;

h) Com base em todas as informações coletadas e solicitadas pelo grupo assessor da comissão, o colegiado da CITEC emitia recomendação ao Ministro da Saúde quanto à incorporação ou não da tecnologia analisada no SUS;

i) As conclusões eram encaminhadas para decisão do Ministro de Estado da Saúde;

j) As decisões eram informadas ao solicitante;

1) As Secretarias efetuavam procedimentos de protocolização no SUS, nos protocolos, diretrizes e manuais.

Para identificação de instrumentos utilizados para caracterizar e avaliar os estudos em ATS realizados no âmbito de organizações responsáveis por seu uso nos processos de análise e decisão para incorporação de tecnologias em saúde foi feita revisão da literatura. Foi selecionada a proposta desenvolvida pela European Network for Health Technology Assessment (EUnetHTA), organização vinculada à Organização Mundial da Saúde (OMS) e que analisa o desempenho das organizações de ATS na Comunidade Europeia 21.

Os principais critérios de qualidade das avaliações de tecnologias em saúde considerados nessa proposta foram:

a) Relevância: temas apropriados definidos pelos decisores políticos abordando tecnologias novas ou em uso.

b) Aplicabilidade: nível de utilização do estudo de ATS pelos decisores políticos respondendo às suas perguntas em relação à tecnologia avaliada. c) Validade: qualidade técnica e metodológica, científica e independência e transparência na elaboração dos estudos de ATS.

d) Oportunidade: finalização dos estudos de ATS em tempo oportuno para as decisões (revisões rápidas, informes de alerta precoce - MHT, ATS para identificar novas pesquisas e ATS completa). 
e) Acessibilidade: disponibilidade pública dos estudos de ATS em base de dados de acesso livre e de fácil localização contendo resumos executivos direcionados aos gestores.

f) Eficiência: impacto sobre os gestores, as práticas, as políticas e os resultados em saúde; equilíbrio entre tempo e qualidade da produção do estudo de ATS; aproveitamento dos recursos disponíveis evitando duplicidade.

g) Equidade: abrangência das tecnologias avaliadas quanto aos propósitos preventivos, diagnósticos, terapêuticos e reabilitadores; resposta a potenciais desigualdades de acesso a grupos sociais; resposta a tópicos relevantes para grupos vulneráveis; promoção da capacidade de utilizar e produzir estudos de ATS em países de media e baixa rendas.

As dimensões de qualidade dos estudos de ATS e dos seus usos nos processos de incorporação de tecnologias dependem, para a sua viabilização, de infraestrutura de pesquisa em geral e em ATS. Dependem também, da existência de múltiplos mecanismos que articulem a produção do conhecimento com as diversas instâncias da gestão dos sistemas de saúde e, principalmente, de compromissos políticos com a sustentabilidade de políticas de saúde que tenham como objetivo o bem-estar da população. O estudo de como ocorrem esses processos concretos permite identificar como os condicionantes maiores se mostram presentes, ou não, na dinâmica cotidiana da gestão de tecnologias.

Na Tabela 1, apresentamos as variáveis selecionadas para o registro das avaliações de tecnologias realizadas pelo DECIT para a CITEC no período de estudo, e que permitem identificar as dimensões de qualidade priorizadas 21 . São variáveis relativamente simples e passíveis de padronização como: datas de entrada da solicitação para análise na CITEC e quem solicitou, doença e especialidade de referência da tecnologia, caracterização da tecnologia, critérios avaliados na ATS, tipo de estudo em ATS realizado, caracterização do estudo e o responsável pela sua realização, duração do estudo, principais conclusões e tipo de decisão sobre a incorporação da tecnologia no SUS.

O trabalho teve como base os dados retrospectivos provenientes de registros e documentos oficiais do período de 2008 a 2010, produzidos pelo DECIT e pela CITEC. Os registros eleitos para a sistematização dos dados foram atas de reunião da comissão, memorandos de solicitação de estudos, registros com as providências do DECIT para a realização das avaliações e a base de dados da REBRATS. Esses registros apresentaram as dificuldades inerentes a toda pesquisa que se baseia em dados secundários e instrumentos que atenderam na sua origem a objetivos específicos diferentes dos da pesquisa. As unidades de análise dos dados selecionadas foram as tecnologias incluídas na pauta da CITEC e as solicitações de estudos ao DECIT. Dois pesquisadores analisaram todas as atas das reuniões da CITEC para obter informações sobre estudos produzidos nas diferentes fontes de dados existentes.

As informações recuperadas para cada tecnologia foram incluídas na planilha desenvolvida para o trabalho. Observou-se que muitas das variáveis eleitas para o estudo não puderam ser recuperadas nas fontes de informação disponíveis, o que levou à necessidade de priorizar na análise as variáveis com maior completude: doença ou agravo segundo capítulos da 10a revisão da Classificação Internacional de Doenças (CID-10); instituição requerente da incorporação, propósito da tecnologia (prevenção, diagnóstico, tratamento, reabilitação); e tipo de estudo elaborado.

Foram produzidos dois tipos de estudos: (1) Nota Técnica de Revisão Rápida (NTRR) - documento de síntese que valoriza revisões sistemáticas, avaliações de tecnologias de agências internacionais e ensaios clínicos, nesta ordem. São estudos produzidos pela equipe interna do DECIT, com tempo de elaboração médio de 10 a 20 dias; e (2) Parecer Técnico Científico (PTC), que consiste em documento de síntese da literatura produzido pela equipe interna ou encomendado a instituições da REBRATS baseado em busca estruturada, inclusão de todos os estudos que atendem a critérios de qualidade e a avaliação crítica de evidências. Todo PTC passava por revisão interna e revisão de consultores pertencentes à REBRATS, especialistas no tema. O tempo médio para entrega do relatório final deveria ser de dois a quatro meses, dependendo do escopo da pergunta e das dimensões da avaliação.

De acordo com os recursos disponíveis, apoiou-se o desenvolvimento de estudos identificados com base no monitoramento de tecnologias emergentes e por mecanismos de priorização junto aos demandantes do Ministério da Saúde. Esses estudos recorreram a diferentes estratégias metodológicas, tais como revisões sistemáticas, avaliações econômicas, análises da gestão de tecnologias em saúde e outros. Em média, foram realizados no prazo de dois a três anos, com análises mais complexas e utilizando dados e informações não disponíveis na rotina habitual.

As tecnologias pautadas na CITEC e analisadas pelo DECIT estão descritas na Tabela 2. Das tecnologias pautadas, muitas foram abordadas mais de uma vez devido ao amplo espectro de indicações. Nos anos considerados, houve tec- 
Tabela 1

Variáveis selecionadas para o estudo de caso, classificadas conforme dimensões de qualidade da avaliação de tecnologias em saúde (ATS).

Categoria

Subcategoria

Critérios de qualidade

de uma ATS

$a$ b $c$ d e $f$

\author{
1. Data de entrada \\ 2. Número do documento \\ 3. Doença/Agravo \\ 4. Tecnologia avaliada \\ 5. Tecnologia avaliada \\ 6. População alvo do estudo \\ 7. Indicação da tecnologia \\ 8. Tipo de demandante \\ 9. Especialidade da tecnologia \\ 10. Propósito da tecnologia avaliada \\ 11. Tipo de tecnologia \\ 12. Padrão ouro \\ 13. Comparador \\ 14. Critérios avaliados
}

15. Tipo de estudo

16. Fase do ciclo de vida da tecnologia

17. Instituição elaboradora

18. Formação do elaborador responsável

19. Tempo de experiência do elaborador responsável

20. Nível de evidência considerada no estudo

21. Especialidade do revisor externo

22. Tempo de experiência do revisar externo

23. Custo do estudo

24. Envolvimento dos tomadores de decisão

25. Envolvimento de serviços e organizações

26. Data de finalização

27. Duração da elaboração do estudo

28. Resumo executivo do estudo

29. Recomendação do estudo

30. Deliberação decisória

31. Grupos de pressão social
Data de entrada da solicitação do estudo

Registro oficial da solicitação do estudo

10ạ revisão da Classificação Internacional de Doenças (CID-10)

Nome genérico

Nome comercial

Não se aplica

Indicação solicitada pelo demandante/interessado

Área que solicitou o estudo

Cardiologia, neurologia, vigilância e controle de agravos etc.

Preventiva, diagnóstica, terapêutica, reabilitadora

Equipamento, vacinas, produtos para saúde, medicamentos, sistema de informação, programas organizacionais

Padrão de referência para o propósito da tecnologia avaliada

Não se aplica

Eficácia, segurança, efetividade, custo-efetividade, custo-utilidade, custo-minimização, custo-benefício, impacto orçamentário, ético, social, judicial, organizacional, ambiental.

Parecer Técnico Científico, Parecer Técnico Científico de Múltiplas Tecnologias, Protocolo Clínico e Diretriz Terapêutica, pesquisa bibliográfica, Nota Técnica de Revisão Rápida, revisão sistemática, avaliação econômica, análise de impacto orçamentário, estudos descritivos, ATS para equipamentos médicos assistenciais.

Inovação, incorporação, difusão inicial, difusão ampla, abandono, exclusão

Anos

Tabela grade

Não se aplica

Anos

Recurso financeiro disponibilizado para o estudo em Reais

Sim, não

Sim, não, não se aplica

Em dias inclui o tempo de revisão externa

Presença ou ausência

Recomendado, recomendado com restrições, não recomendado, recomendado exclusão

Incorporado para a indicação, incorporação com restrição, não incorporação para a indicação, exclusão, ampliação de CID-10

Sociedade de pacientes, judiciário, indústria, gestores
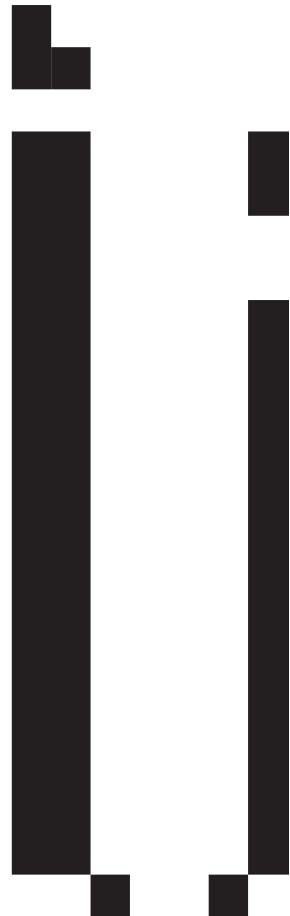

a: relevância; b: aplicabilidade; c: validade; d: oportunidade; e: acessibilidade; f: eficiência; g: equidade. 
Participação do Departamento de Ciência e Tecnologia (DECIT) na Comissão de Incorporação de Tecnologias (CITEC), Brasil: tecnologias pautadas, analisadas e suas características principais de 2008 a 2010.

\begin{tabular}{|c|c|c|c|c|}
\hline Temas avaliados & 2008 & 2009 & 2010 & Total \\
\hline \multicolumn{5}{|l|}{ Tecnologias pautadas na CITEC e analisadas pelo DECIT } \\
\hline Tecnologias pautadas & 9 & 25 & 69 & 103 \\
\hline Tecnologias analisadas pelo DECIT & 7 & 18 & 47 & 72 \\
\hline Percentual de tecnologias analisadas & $78 \%$ & $72 \%$ & $68 \%$ & $70 \%$ \\
\hline Tecnologias que permaneceram em análise & 1 & 1 & 4 & 6 \\
\hline \multicolumn{5}{|l|}{ Propósitos das tecnologias avaliadas } \\
\hline Tecnologias preventivas & 0 & 1 & 4 & 5 \\
\hline Tecnologias diagnósticas & 0 & 2 & 10 & 12 \\
\hline Tecnologias terapêuticas & 9 & 22 & 54 & 85 \\
\hline Tecnologias reabilitadoras & 0 & 0 & 1 & 1 \\
\hline \multicolumn{5}{|l|}{ Tipos de estudos produzidos } \\
\hline Atualização da Nota Técnica de Revisão Rápida & 0 & 1 & 0 & 1 \\
\hline Atualização de Parecer Técnico Científico & 0 & 4 & 2 & 6 \\
\hline Pesquisa bibliográfica & 0 & 0 & 1 & 1 \\
\hline Análise econômica & 0 & 10 & 2 & 12 \\
\hline Análise de impacto orçamentário & 1 & 0 & 0 & 1 \\
\hline Nota técnica de revisão rápida & 5 & 11 & 20 & 36 \\
\hline Outros * & & 2 & 6 & 8 \\
\hline Protocolo Clínico e Diretriz Terapêutica & 1 & 1 & 14 & 16 \\
\hline Parecer Técnico Científico & 3 & 4 & 19 & 26 \\
\hline Parecer Técnico Científico de Múltiplas Tecnologias ** & 0 & 0 & 1 & 1 \\
\hline Revisão sistemática & 0 & 4 & 1 & 5 \\
\hline \multicolumn{5}{|l|}{ Estudos produzidos por instituição } \\
\hline DECIT & 9 & 18 & 23 & 50 \\
\hline DECIT e Grupo Protocolo Clínico e Diretriz Terapêutica & & 1 & 1 & 2 \\
\hline DECIT e REBRATS & & 1 & & 1 \\
\hline SAS e Grupo Protocolo Clínico e Diretriz Terapêutica & 1 & & 17 & 18 \\
\hline REBRATS & & 17 & 23 & 40 \\
\hline SVS & & & 2 & 2 \\
\hline \multicolumn{5}{|l|}{ Demandantes de estudos de tecnologias para incorporação no } \\
\hline \multicolumn{5}{|l|}{ SUS } \\
\hline CITEC & 7 & 9 & 23 & 39 \\
\hline Departamento de Assistência Farmacêutica & & & 2 & 2 \\
\hline DECIT & & 6 & 13 & 19 \\
\hline DECIT 2003 e CITEC & & & 7 & 7 \\
\hline Ministério da Saúde/FUNED & & & 1 & 1 \\
\hline SAS & & 7 & 19 & 26 \\
\hline SAS (CITEC) & & & 1 & 1 \\
\hline SVS & 2 & 3 & 3 & 8 \\
\hline
\end{tabular}

FUNED: Fundação Ezequiel Dias; REBRATS: Rede Brasileira de Avaliação de Tecnologias em Saúde; SAS: Secretaria de Atenção à Saúde; SVS: Secretaria de Vigilância em Saúde.

* Manual de Vigilância da Leishmaniose, Comitê do Programa DST/AIDS, Opinião de especialista, Revisão de Protocolo Clínico e Diretriz Terapêutica e Estudo de Eficácia e Segurança;

** Neste estudo foram avaliadas nove tecnologias para feridas e queimaduras. 
nologia que permaneceu em análise de 2007 para 2008 e de 2008 para 2009, e quatro tecnologias estavam em análise no fim de 2010.

Em relação à CID-10 houve maior frequência de tecnologias pautadas para doenças endócrinas, nutricionais e metabólicas (17/103), com ênfase em terapêutica de doenças metabólicas raras e dislipidemias. As doenças infecciosas e parasitárias tiveram a segunda maior frequência (16/103), com foco na terapêutica em hepatites virais e AIDS. As neoplasias ocuparam o terceiro lugar com 15 das 103 tecnologias pautadas.

As características das tecnologias pautadas na CITEC, nos anos avaliados, estão apresentadas na Tabela 2, verificando-se que aproximadamente $82 \%$ tinham atuação terapêutica $(85 / 103)$. Isso se deve à predominância de tecnologias com alto interesse do mercado, de média e alta complexidades e à valorização de cuidados hospitalares e especializados, mais intensivos em tecnologias de produto 23 .

A maior parte dos estudos foi conduzida pelo DECIT, totalizando 82\% (DECIT e REBRATS) dos trabalhos produzidos entre 2008 e 2010. Em relação aos processos de produção, alguns foram realizados pela equipe interna do DECIT e outros por instituições da REBRATS contratadas.

Foi produzido um total de 103 estudos para auxiliar as decisões no âmbito da CITEC. Em relação ao tipo de estudos houve predominância do NTRR (36/103) e PTC (26/103). Observou-se uma produção crescente do número de estudos realizados pelo DECIT e instituições vinculadas à REBRATS, conforme mostra a Tabela 2. Esse fato pode ser explicado pela formalização do papel do DECIT na CITEC a partir do final de 2008, proporcionando um aumento da equipe do DECIT nesses anos, e pela consolidação da REBRATS. Entre 2008 para 2010, o incremento proporcional foi de $61 \%$ para o DECIT e em um ano houve um incremento de $26 \%$ de estudos da REBRATS, representando aumento da demanda para a rede.

As demandas por estudos ao DECIT pelo Ministério da Saúde eram originárias de vários departamentos, sendo a CITEC a maior demandante $(38 \%)$ juntamente com a SAS (25\%). O DECIT também antecipou o estudo de temas a serem avaliados, representando 19\% das avaliações, como mostra a Tabela 2.

Em virtude da inconsistência dos registros encontrados, foi inviabilizada a análise de qualidade dos estudos produzidos e a concordância entre os resultados e recomendações dos estudos e as decisões da CITEC. Mas, apesar de não ser possível a análise da qualidade dos estudos produzidos ao longo do tempo, o aumento na produção de NTRR e de pareceres técnico-científicos é positivo. São estudos de síntese, elaborados com relativa rapidez, para atender à pauta de deliberação, e que anteriormente dependiam quase que exclusivamente de opiniões de especialistas. A relação entre tempo e qualidade da síntese e do conhecimento produzido é bastante discutida quando se exigem prazos curtos para deliberação ${ }^{24}$. Há necessidade de aprimorar o desenvolvimento de bons métodos para a elaboração de respostas rápidas, que atendam às necessidades dos tomadores de decisão no horizonte temporal exigido, sem perder de vista o cuidado que deve existir nas recomendações sobre a utilização de tecnologias em condições de assistência geralmente ainda pouco estudadas.

\section{Considerações finais}

Fica clara a necessidade de implantação de registros estruturados para que seja possível analisar padrões de qualidade e impactos da ATS, e atender às demandas crescentes por transparência, confiabilidade e consistência que diferentes setores econômicos e sociais colocam aos responsáveis pelas decisões de incorporação de tecnologias. Neste trabalho, ainda que de forma restrita, os dados apresentados permitem identificar que no período houve progressiva estruturação de processos mais consistentes na realização e utilização de estudos de ATS nos processos de análise para incorporação de tecnologias no SUS. A caracterização dos estudos e processos é etapa importante na construção de proposta de base analítica que poderia contribuir para o monitoramento dos processos de ATS desenvolvidos no Ministério da Saúde.

O DECIT atuou ativamente na CITEC do Ministério da Saúde produzindo a maioria das avaliações, conforme previsto nas normas vigentes no período. A REBRATS teve papel crescente na produção dos estudos.

Não foi possível analisar a qualidade dos estudos produzidos, assim como o grau de impacto nas decisões ou a concordância entre os resultados e recomendações dos estudos nas decisões da CITEC, e esta é uma pergunta crucial ainda a ser respondida.

Recentemente, foram identificados alguns dos desafios para que o campo da avaliação de tecnologias tenha impactos no sistema de saúde brasileiro, destacando-se a necessidade de ampliação do foco para ações de retirada de tecnologias, intersecção com gestão estadual, municipal e saúde suplementar, autonomia e transparência nos processos institucionais, assim como imprimir ação ativa na definição de prioridades e na influência do mercado privado 25 . 
No Brasil, como em todos os países em desenvolvimento, o processo de aprendizado tanto metodológico quanto político está em curso e merece sucessivas reavaliações. Em que pese os avanços na regulação da incorporação de tecno- logias em saúde no SUS, permanece a discussão sobre como enfrentar os desafios para a gestão de um sistema único, igualitário e universal tal como proposto na Constituição Federal.

\section{Resumen}

Las políticas científicas con objetivos de producción de conocimiento en materia de salud se han ampliado en las últimas décadas. En Brasil, la promoción de la investigación, el desarrollo y la innovación en materia de salud forma parte de la Constitución desde 1990, y se han propuesto políticas de ciencia y tecnología para la salud desde 1994, incluyendo políticas de desarrollo de la evaluación de tecnologías sanitarias (ETS), como en otros países. Se trata ahora de evaluar el impacto de la ETS en la gestión y la incorporación de las tecnologías a los sistemas de salud. Describimos como estudio de caso la utilización de los procesos de ETS para incorporar tecnologías en Brasil y se analizó la participación del Departamento de Ciencia y Tecnología (DECIT) del Ministerio de Salud en la Comisión de Incorporación de Tecnología del Ministerio de Salud (CITEC), durante el período 2008-2010. Se utilizaron 103 estudios del CITEC, un 80\% producido por el DECIT, la mayoría de sintesis basadas en la literatura existente, el $80 \%$ fueron tecnologías con fines terapéuticos, con una tendencia de crecimiento de este tipo producción científica durante el periodo. Hay un proceso de aprendizaje continuo para el uso metodológico y político del ETS, pero hay una necesidad de análisis adicionales sobre su impacto en el Sistema Único de Salud.

Sistema Único de Salud; Estudios de Evaluación;

Evaluación de Tecnologías de Salud

\section{Colaboradores}

H. M. D. Novaes trabalhou no delineamento do estudo e redação final do artigo. F. T. S. Elias participou no delineamento do estudo, na coleta, análise e interpretação dos dados e na redação das versões do artigo.

\section{Conflito de interesses}

Não declarado. 


\section{Referências}

1. Guimarães R. Pesquisa em saúde no Brasil. Rev Saúde Pública 2006; 40(n. esp.):3-10.

2. Gadelha CAG. Desenvolvimento, complexo industrial da saúde e política industrial. Rev Saúde Pública 2006; 40(n. esp.):11-23.

3. Guimarães R. Pesquisa translacional: uma interpretação. Ciênc Saúde Coletiva 2013; 18:1731-44.

4. Ward V, House A, Hamer S. Developing a framework for transferring knowledge into action: a thematic analysis of the literature. J Health Serv Res Policy 2009; 14:156-64.

5. Buxton M, Hanney S, Jones T. Estimating the economic value to societies of the impact of health research: a critical review. Bull World Health Organ 2004; 82:733-6.

6. Coordenação Geral de Desenvolvimento em Ciência e Tecnologia, Ministério da Saúde. Anais da I Conferência Nacional de Ciência, Tecnologia em Saúde - I CNCTS. Brasília: Ministério da Saúde; 1994.

7. Banta HD, Luce BR. Health care technology and its assessment: an international perspective. New York: Oxford University Press; 1993.

8. Karam RAS. Modernização gerencial: um caminho obrigatório para a consolidação e fortalecimento do Sistema Único de Saúde: a experiência do Projeto REFORSUS [Dissertação de Mestrado]. Rio de Janeiro: Escola Brasileira de Administração Pública, Fundação Getúlio Vargas; 2002.

9. Silva LK. Avaliação tecnológica e avaliação de custo-efetividade em saúde: a incorporação de tecnologias e a produção de diretrizes clínicas para o SUS. Ciênc Saúde Coletiva 2003; 8:501-20.

10. Banta R, Almeida RT. The development of health technology assessment in Brazil. Int J Technol Assess Health Care 2009; 25 Suppl 1:255-9.

11. Secretaria de Ciência, Tecnologia e Insumos Estratégicos, Ministério da Saúde. Política Nacional de Gestão de Tecnologias em Saúde. Brasília: Ministério da Saúde; 2011.

12. Departamento de Ciência e Tecnologia, Secretaria de Ciência, Tecnologia e Insumos Estratégicos, Ministério da Saúde. Encontro anual da Sociedade Health Technology Assessment International, Brasil, 2011. Rev Saúde Pública 2011; 45:1201-5.

13. Garrido MV, Kristensen FB, Nielsen CP, Busse R. Health technology assessment and health policymaking in Europe current status: challenges and potential. Copenhagen: European Observatory on Health Systems and Policies/WHO Regional Office for Europe; 2008. (Observatory Studies, 14).

14. Schwarzer R, Siebert U. Methods, procedures and contextual characteristics of health technology assessment and health policy decision making: comparison of health technology assessments agencies in Germany, United Kingdom, France and Sweden. Int J Technol Assess Health Care 2009; 25:305-14.
15. Neumann PJ, Drummond MF, Jonsson B, Luce BR, Schwartz JS, Siebert U, et al. Are key principles for improved health technology assessment supported and used by health technology assessment organizations? Int J Technol Assess Health Care 2010; 26:71-8.

16. Pichon-Riviere A, Augustovski F, Rubinstein A, Marti SG, Sullivan SD, Drummond MF. Health technology assessment for resource allocation decisions: are key principles relevant for Latin America? Int J Technol Assess Health Care 2010; 26:421-7.

17. Arellano LE, Reza M, Blasco JA, Andradas E. A content analysis of health technology assessment programs in Latin America. Int J Technol Assess Health Care 2009; 25:570-6.

18. Moharra M, Kubesch N, Estrada MD, Parada A, Cortés M, Espallargues M on behalf of Work Package 8, EUnetHTA project. Survey report on HTA organisations. Barcelona: Catalan Agency for Health Technology Assessment and Research, Catalan Health Service, Department of Health, Autonomous Government of Catalonia; 2008.

19. Wilsdon TIM, Serota AMY. A comparative analysis of the role and impact of health technology assessment. London: Charles River Associates; 2011. (CRA Project, D15891-00).

20. Oliver A, Mossialos E, Robinson R. Health technology assessment and its influence on health-care priority setting Int J Technol Assess Health Care 2004; 20:1-10.

21. Kristensen FB, Lampe K, Chase DL, Lee-Robin SH, Wild C, Moharra M, et al. Practical tools and methods for health technology assessment in Europe: structures, methodologies and tools developed by the European network for Health Technology Assessment, EUnHTA. Int J Technol Assess Health Care 2009; 25 Suppl 2:1-8.

22. Elias FTS, Silveira DG, Novaes HMD. Avaliação de tecnologias em saúde no Ministério da Saúde do Brasil: processos de análise e tomada de decisão: relatório final. Brasília: Departamento de Ciência e Tecnologia, Secretaria de Ciência e Tecnologia e Insumos Estratégicos, Ministério da Saúde; 2012.

23. Giovanella L, Escorel S, Lobato LVC, Noronha JC, Carvalho AI, organizadores. Políticas e sistema de saúde no Brasil. Rio de Janeiro: Editora Fiocruz; 2008.

24. Milne R, Clegg A, Stevens A. HTA responses and the classic HTA report. J Public Health Med 2003; 25:102-6.

25. Hudson PS, Petramale CA, Elias FTS. Avanços e desafios da política nacional de gestão de tecnologias e saúde. Rev Saúde Pública 2012; 46:83-90.

Recebido em 24/Jan/2013

Versão final reapresentada em 20/Ago/2013 Aprovado em 27/Ago/2013 